\title{
Laser-induced breakdown measurements of silica nanofibers in air and immersed in water, ethanol and isopropanol
}

\author{
Maha Bouhadida ${ }^{1 *}$ and Sylvie Lebrun ${ }^{1}$ \\ ${ }^{1}$ Laboratoire Charles Fabry, Institut d'Optique, CNRS, Université Paris-Saclay, 91127 Palaiseau cedex, France
}

\begin{abstract}
In this paper we present the first study to our knowledge of Laser-Induced Breakdown (LIB) measurements of silica nanofibers in air and in three liquids for different radii in the ns regime. The experimental protocol is described. A significant number of samples are tested and the results are highly repeatable. We showed that immerging a nanofiber in a liquid substantially enhanced the LIB, the most salient increase having been obtained for nanofibers immersed in water for which the LIB has been almost multiplied by a factor of 2 compared with air. This property offers a new degree of freedom to widen the field of applications of nanofibers, where high peaks powers are needed.
\end{abstract}

\section{Introduction}

Optical tapered nanofibers are fabricated by pulling standard silica fibers until their diameters become typically smaller than $1 \mu \mathrm{m}$. The nanofibers are linked to the untapered end-fibers via two flared sections called the tapers and can be inserted in fibered networks with very low losses, making them highly attractive for many applications. At such small diameters, nanofibers exhibit a strong confinement of light inside the silica which enables the generation of nonlinear effects [1] such as, for example, the generation of supercontinuum [2]. Nanofibers can also exhibit an intense evanescent field which has been exploited for optical sensing [3] or optical traps for quantum applications [4,5]. The excitation of optical non linearities such as stimulated Raman effect [6,7] or Kerr effect in the evanescent field of nanofibers immersed in liquids has also been demonstrated [8]. In all the above-mentioned applications the optical mode is guided by total internal reflection at the interface between the silica nanofiber and the surrounding medium that can be vacuum, a gas or a liquid. The Laser-Induced Breakdown (LIB) for applications in general and consequently for nanofibers is often a limiting issue. For pulsed regimes, to our knowledge, there is a lack of experimental data on the LIB in nanofibers in the literature.

In this work our aim is to give experimental data and reliable trends on the LIB of silica nanofibers in ambiant air and immersed in different low-index common liquids. We present the experimental protocol we have followed to measure the LIB. The pump source is a sub-ns frequency doubled Nd:YAG microlaser emitting at 532 $\mathrm{nm}$ with a frequency rate repetition of $4.7 \mathrm{kHz}$. The results we obtained are highly repeatable and physical interpretations will be proposed during the conference. We show that immerging a nanofiber in water can increase the LIB obtained in air by more than a factor of 2, offering a simple solution to increase the LIB for many applications in which high peak powers are needed (Raman converters, sensors etc ...).

\section{Experimental setup and protocol}

The experimental setup is depicted in Fig. 1. The preparation and the drawing of the nanofibers were performed in a class-5 clean room. In the following experiments, we have used the SMF28 fiber (ref. Corning). As this fiber is highly multimode at $532 \mathrm{~nm}$, high-order modes were filtered beforehand by pulling the input connecting fiber over $1 \mathrm{~cm}$ until reaching a core radius of $1.3 \mu \mathrm{m}$ and a cladding radius of $20 \mu \mathrm{m}$ allowing the propagation of the fundamental mode only (single mode filter on Fig. 1). We have used short and straight connecting fibers to remain on a single mode propagation from the input (indicated by $\mathrm{E}_{1}$ ) to the output (indicated by $\mathrm{E}_{\text {out }}$ ). Then, before being pulled the fiber was removed from its polymer cladding with a mechanical stripper and cleaned in a single pass with an optical paper wet by a drop of ethanol. The nanofibers were drawn with a pulling rig using the "pull and brush" technique and a simple butane flame to soften the glass [6]. Our pulling rig enabled to routinely draw high optical quality nanofibers with an overall optical transmission $\mathrm{T}$ defined as the ratio of the output energy $E_{\text {out }}$ versus the input energy $E_{1}$ always higher than $80 \%$. Once drawn, the nanofiber was transported out of the clean room and put in the experimental setup in a tank with a removable protection glass cover. The pump source beam, which was linearly polarized, (ref. Horus HLX-G-F004) was sent on a half-wavelength plate associated with a polarizer cube to control the incident energy on the nanofiber. The rejected beam served as a reference for the input energy $\left(\mathrm{E}_{\mathrm{ref}}\right)$. The direct beam was

Corresponding author: maha.bouhadida@institutoptique.fr 
launched in the SMF28 fiber through a microscope objective, propagated across the single mode filter, the nanofiber and was collected at the output for energy measurements. The input energy $E_{1}$ just after the single mode filter was calibrated with respect to $\mathrm{E}_{\text {ref. }}$ The nanofiber was a little bit stretched to be straight but not constrained and kept in the position thanks to two smooth magnets. The pump beam was injected in the fiber at low energy and the optical transmission was optimized. For experiments with liquids, the tank was filled carefully and immediately afterwards with a micro-syringe until the nanofiber was fully immersed. The optical transmission was optimized again and the laser input energy was slowly increased until the nanofiber breakdown. Input and output energies were recorded during all the process.

Four external media were used : ambiant air, tap water, ethanol and isopropanol. The radii of the three nanofibers we tested (NF1, NF2 and NF3) were respectively $220 \mathrm{~nm}, 300 \mathrm{~nm}$ and $350 \mathrm{~nm}$. For each medium we have drawn 10 nanofibers of each radius. The length of each nanofiber was $2 \mathrm{~cm}$ in order to avoid non linear effects such as Raman effect in the liquid and hence to study the LIB alone $[6,7]$.

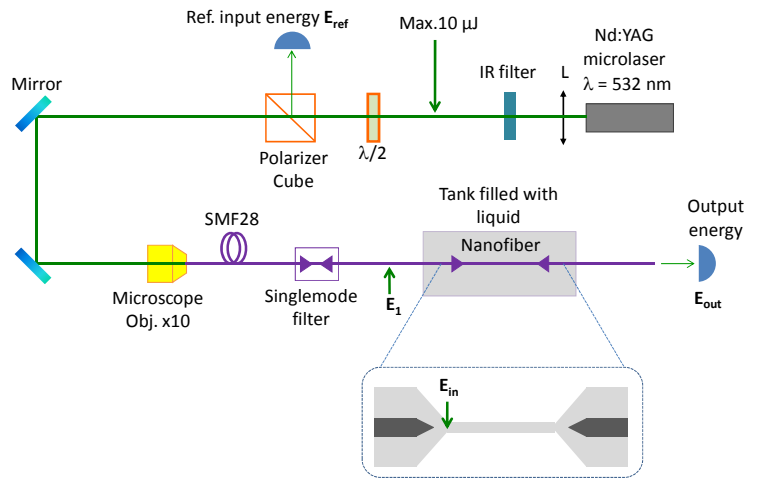

Fig. 1. Experimental setup.

\section{Results}

The overall transmission $\mathrm{T}$ of the immersed nanofibers remained very stable during the whole experiment until the LIB, respectively $90 \%$ in water, $92 \%$ in ethanol and $95 \%$ in isopropanol. This was not the case when they were in air : the average transmission decreased from $80 \%$ to $57 \%$ during the experiment. This first systematic observation showed that whatever the liquid we used it had a protection effect from additional dust. It also enabled to mechanically stabilize the nanofiber, avoiding the fluctuations due to micro-turbulences.

We have plotted in Fig. 2 the energy inside the nanofiber at LIB, named $E_{\mathrm{in}}$, for each radius $\mathrm{r}_{\mathrm{NF}}$ and each external medium :

$$
E_{\text {in }}\left(r_{N F}, \text { medium }\right)=\eta \sqrt{T} E_{1}\left(r_{N F}, \text { medium }\right)
$$

$\eta$ is the fractional energy inside the nanofiber. The standard deviation on the incident energies (not shown on the graphic) was estimated to be $0.01 \mu \mathrm{J}$ in all cases. In this range of radii, the uncertainty on the radii was below $15 \mathrm{~nm}$. The analyze of the results confirmed that surface effects were predominant compared to bulk effects. Fig. 2 shows that immerging a nanofiber in a liquid substantially enhanced the LIB, the most salient increase having been obtained for nanofibers immersed in water for which the LIB has been almost multiplied by a factor of 2 compared with air.

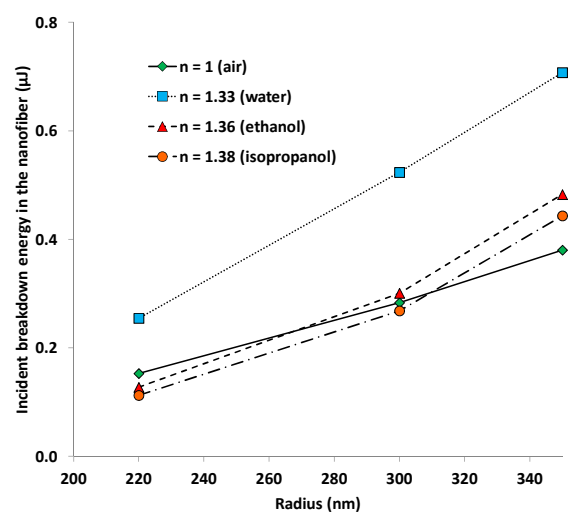

Fig. 2. LIB energy measurements for three nanofiber radii and four external media.

\section{Conclusion}

In this paper we have presented the first study to our knowledge of LIB of nanofibers in air and in several liquids for different radii in the ns regime. The same protocol was applied to a significant number of samples and showed highly repeatable results. We showed that immerging a nanofiber in a liquid substantially enhanced the LIB, offering a new degree of freedom to widen the field of applications of nanofibers, where high peaks powers are needed.

This work is supported by the French National Research Agency (ANR) (FUNFILM-ANR-16-CE24-0010- 03).

\section{References}

[1] M. A. Foster, A.C. Turner, M. Lipson, A.L. Gaeta, Opt. Express 16, pp. 1300-1320 (2008).

[2] S. Leon-Saval, T. Birks, W. Wadsworth et P. Russell, Opt. Express, 12 (13), pp. 2864-2869 (2004).

[3] L. Tong, Sensors, 18, 903 (2018).

[4] B. Gouraud, D. Maxein, A. Nicolas, O. Morin, and J. Laurat, Phys. Rev. Lett. 114, 180503, May 2015.

[5] K. S. Rajasree, T. Ray, K. Karlsson, J. L. Everett, and S. Nic Chormaic, Phys. Rev. Research 2, 012038(R) February 2020.

[6] L. Shan, G. Pauliat, G. Vienne, L. Tong, and S. Lebrun, Applied Physics Letters, 102:201110, May 2013.

[7] M. Bouhadida, J. C. Beugnot, P. Delaye, K. Phan Huy and S. Lebrun, Applied Physics B, Volume 125, Issue 12, pp.1-7 (sept. 2019).

[8] G. Fanjoux, J. Chrétien, A. Godet, K. Phan-Huy, J.C. Beugnot, T. Sylvestre, Opt. Express 27 (20), pp. 29460-29470 (2019). 